\title{
Prevalence and Postdischarge Outcomes Associated with Frailty in Medical Inpatients: Impact of Different Frailty Definitions
}

\author{
Finlay A McAlister, MD, MSc ${ }^{1,2 *}$; Mu Lin, PhD2; Jeffrey A Bakal, PhD, PStat ${ }^{2}$
}

${ }^{1}$ Division of General Internal Medicine, University of Alberta, Edmonton, Alberta, Canada; ${ }^{2}$ Alberta SPOR Support Unit Data Platform, University of Alberta, Edmonton, Alberta, Canada.

We compared prevalence estimates and prognostication if frailty were defined using the face-to-face Clinical Frailty Scale (CFS) or the administrative-data-derived Hospital Frailty Risk Score (HFRS). We evaluated 489 adults from a prospective cohort study of medical patients being discharged back to the community; 276 (56\%) were deemed frail (214 [44\%] on the HFRS and 161 [33\%] on the CFS), but only 99 (20\%) met both frailty definitions (kappa $0.24,95 \% \mathrm{Cl} 0.16-0.33)$. Patients classified as frail on the CFS exhibited significantly higher 30-day readmission/ death rates, $19 \%$ versus $10 \%$ for those not frail (aOR [adjusted odds ratio] 2.53, 95\% Cl 1.40-4.57) and 21\% versus $6 \%$ for those aged $>65$ years (aOR $4.31,95 \% \mathrm{Cl}$ 1.80-10.31). Patients with HFRS-defined frailty exhibited higher 30-day readmission/death rates that were not statistically significant (16\% vs $11 \%$, aOR 1.62 [95\% C $0.95-2.75]$ in all adults and $14 \%$ vs $11 \%$, aOR 1.24 [95\% Cl $0.58-2.83$ ] in those aged $>65$ years). Journal of Hospital Medicine 2019;14:407-410. Published online first March 20, 2019. (C) 2019 Society of Hospital Medicine

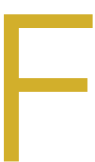
railty is associated with adverse outcomes in hospitalized patients, including longer length of stay, increased risk of institutionalization at discharge, and higher rates of readmissions or death postdischarge. ${ }^{1-4}$ Multiple tools have been developed to evaluate frailty and in an earlier study, ${ }_{1}^{4}$ we compared the three most common of these and demonstrated that the Clinical Frailty Scale $(C F S)^{5}$ was the most useful tool clinically as it was most strongly associated with adverse events in the first 30 days after discharge. However, it must be collected prospectively and requires contact with patients or proxies for the evaluator to assign the patient into one of nine categories depending on their disease state, mobility, cognition, and ability to perform instrumental and functional activities of daily living. Recently, a new score has been described which is based on an administrative data algorithm that assigns points to patients having any of 109 ICD-10 codes listed for their index hospitalization and all hospitalizations in the prior two years and can be generated retrospectively without trained observers. ${ }^{6}$ Although higher Hospital Frailty Risk Scores (HFRS) were associated with greater risk of postdischarge adverse events, the kappa when compared with the CFS was only 0.30 (95\% $\mathrm{Cl} 0.22-0.38$ ) in that study. ${ }^{6}$ However, as the HFRS was developed and validated in patients aged $\geq 75$ years within the UK National Health Service, the authors them-

\footnotetext{
*Corresponding Author: Finlay A McAlister, MD, MSc; E-mail: Finlay.McAlister@ualberta.ca; Telephone: 780-492-9824.

Additional Supporting Information may be found in the online version of this article.
}

Received: December 6, 2018; Revised: January 23, 2018;

Accepted: January 27, 2018

@2019 Society of Hospital Medicine DOI 10.12788/jhm.3174 selves recommended that it be evaluated in other healthcare systems, other populations, and with comparison to prospectively collected frailty data from cumulative deficit models such as the CFS

The aim of this study was to compare frailty assessments using the CFS and the HFRS in a population of adult patients hospitalized on general medical wards in North America to determine the impact on prevalence estimates and prediction of outcomes within the first 30 days after hospital discharge (a timeframe highlighted in the Affordable Care Act and used by Centers for Medicare \& Medicaid Services as an important hospital quality indicator).

\section{METHODS}

As described previously, ${ }^{7}$ we performed a prospective cohort study of adults without cognitive impairment or life expectancy less than three months being discharged back to the community (not to long-term care facilities) from general medical wards in two teaching hospitals in Edmonton, Alberta, between October 2013 and November 2014. All patients provided signed consent, and the University of Alberta Health Research Ethics board (project ID Pro00036880) approved the study.

Trained observers assessed each patient's frailty status within 24 hours of discharge based on the patient's best status in the week prior to becoming ill with the reason for the index hospitalization. The research assistant classified patients into one of the following nine CFS categories: very fit, well, managing well, vulnerable, mildly frail (need help with at least one instrumental activities of daily living such as shopping, finances, meal preparation, or housework), moderately frail (need help with one or two activities of daily living such as bathing and dressing), severely frail (dependent for personal care), very severely frail (bedbound), and terminally ill. According to the CFS 
TABLE 1. Baseline Characteristics of Cohort Patients

\begin{tabular}{|c|c|c|c|c|c|}
\hline & $\begin{array}{l}\text { Not Frail on CFS or HFRS } \\
\text { Models, } n=213\end{array}$ & $\begin{array}{l}\text { Frail on the CFS only, } \\
\qquad n=62\end{array}$ & $\begin{array}{l}\text { Frail on the HFRS only, } \\
\qquad n=115\end{array}$ & $\begin{array}{l}\text { Frail on CFS and HFRS, } \\
\qquad n=99\end{array}$ & $\begin{array}{l}P \text { Value Comparing } \\
\text { the Columns }\end{array}$ \\
\hline Age, y, mean $(95 \% \mathrm{Cl})$ & $57.8(55.4,60.2)$ & $73.8(70 . .0,77.7)$ & $61.4(57.9,64.8)$ & $72.6(69.7,75.5)$ & $<.01$ \\
\hline Sex, female, no (\%) & $95(44.6)$ & $39(62.9)$ & $48(41.7)$ & $64(64.7)$ & $<.01$ \\
\hline No. of comorbidities, mean $(95 \% \mathrm{Cl})$ & $3.6(3.3,3.9)$ & $6.0(5.3,6.6)$ & $5.3(4.8,5.8)$ & $6.4(5.9,7.0)$ & .01 \\
\hline Charlson comorbidity score, mean (95\% Cl) & $2.0(1.7,2.2)$ & $3.2(2.7,3.7)$ & $3.3(2.9,3.8)$ & $3.8(3.4,4.2)$ & .16 \\
\hline No. of patients hospitalized in prior 12 months, no (\%) & $47(22.1)$ & $23(37.1)$ & $72(62.6)$ & $73(73.7)$ & $<.01$ \\
\hline Preadmission living situation, no (\%) & & & & & $<.01$ \\
\hline Living at home independently & $169(79.3)$ & $20(32.3)$ & $73(63.5)$ & $23(23.2)$ & \\
\hline Living at home with help & $43(20.2)$ & $35(56.5)$ & $34(29.6)$ & $54(54.6)$ & \\
\hline Assisted living or lodge & $1(0.5)$ & $7(11.3)$ & $8(7.0)$ & $22(22.2)$ & \\
\hline EQ-5D overall score, /100, mean $(95 \% \mathrm{CI})$ & $65.7(63.4,68.1)$ & $60.7(55.6,65.9)$ & $65.1(61.8,68.4)$ & $60.0(54.8,63.2)$ & .06 \\
\hline Goals of care in the hospital, no (\%) Resuscitation/ICU & & & & & $<.01$ \\
\hline ICU but no resuscitation & $179(87.8)$ & $26(47.3)$ & $86(77.5)$ & $43(46.7)$ & \\
\hline No ICU, no resuscitation & $13(6.4)$ & $12(21.8)$ & $9(8.1)$ & $21(22.8)$ & \\
\hline \multirow[t]{2}{*}{ Comfort care } & $12(5.9)$ & $17(30.9)$ & $15(13.5)$ & $28(30.4)$ & \\
\hline & $0(0)$ & $0(0)$ & $1(0.9)$ & $0(0)$ & \\
\hline Timed Up and Go Test, s, mean $(95 \% \mathrm{Cl})$ & $12.9(11.2,14.7)$ & $23.2(18.9,27.5)$ & $13.2(12.1,14.3)$ & $25.4(21.9,28.9)$ & $<.01$ \\
\hline Grip Strength, KG, mean (95\% Cl) & $31.8(30.1,33.5)$ & $12.8(19.6,24.0)$ & $28.5(26.4,30.6)$ & $20.0(18.3,21.6)$ & $<.01$ \\
\hline Serum albumin, g/L, mean $(95 \% \mathrm{Cl})$ & $35.0(33.5,36.5)$ & $35.8(33.6,38.0)$ & $31.5(29.6,33.4)$ & $33.0(31.5,34.5)$ & $<.01$ \\
\hline $\begin{array}{l}\text { No. of prescription medications at discharge, } \\
\text { mean }(95 \% \mathrm{CI})\end{array}$ & $4.6(4.1,5.0)$ & $9.0(8.1,9.9)$ & $6.0(5.4,6.6)$ & $8.2(7.5,8.9)$ & $<.01$ \\
\hline Length of stay, d, median, [IQR] & $7.2(3.8,10.7)$ & $6.9(5.4,8.4)$ & $7.6(6.6,8.6)$ & $10.4(8.5,12.5)$ & $<.01$ \\
\hline
\end{tabular}

Definitions of frailty: scoring $\geq 5$ on the Clinical Frailty Scale (CFS), $\geq 5$ on the Hospital Frailty Risk Score (HFRS)

validation studies, the last five categories were defined as frail for the purposes of our analyses.

Independent of the trained observer's assessments, we calculated the HFRS for each participant in our cohort by linking to Alberta administrative data holdings within the Alberta Health Services Data Integration and Measurement Reporting unit and examining all diagnostic codes for the index hospitalization and any other hospitalizations in the prior two years for the 109 ICD-10 codes listed in the original HFRS paper and used the same score cutpoints as they reported (HFRS $<5$ being low risk, 5-15 defined as intermediate risk, and $>15$ as high risk for frailty; scores $\geq 5$ were defined as frail). ${ }^{6}$

All patients were followed after discharge by research personnel blinded to the patient's frailty assessment. We used patient/caregiver self-report and the provincial electronic health record to collect information on all-cause readmissions or mortality within 30 days.

We have previously reported ${ }^{4,7}$ the association between frailty defined by the CFS and unplanned readmissions or death within 30 days of discharge but in this study, we examined the correlation between CFS-defined frailty and the HFRS score (classifying those with intermediate or high scores as frail) using chance-corrected kappa coefficients. We also compared the prognostic accuracy of both models for predicting death and/or unplanned readmissions within 30 days using the $C$ statistic and the integrated discrimination improvement index and examined patients aged $>65$ years as a subgroup. ${ }^{8}$ We used SAS version 9.4 (SAS Institute, Cary, North Carolina) for analyses, with $P$ values of $<.05$ considered as statistically significant.

\section{RESULTS}

Of the 499 patients in our original cohort, ${ }^{7}$ we could not link 10 to the administrative data to calculate HFRS, and thus this study sample is only 489 patients (mean age 64 years, 50\% women, $52 \%$ older than 65 years, a mean of 4.9 comorbidities, and median length of stay five days).

Overall, 276 (56\%) patients were deemed frail according to at least one assessment (214 [44\%] on the HFRS [35\% intermediate risk and $9 \%$ high risk] and 161 [33\%] on the CFS), and 99 (20\%) met both frailty definitions (Appendix Figure). Among the 252 patients aged $>65$ years, 66 (26\%) met both frailty 
TABLE 2. Predictive Ability of Different Frailty Assessment Methods Adjusted for Age and Sex

\begin{tabular}{|c|c|c|c|}
\hline Frailty Definition Met & $\begin{array}{l}\text { Adjusted Odds Ratio for 30-Day } \\
\text { Readmission/Death }\end{array}$ & $95 \% \mathrm{Cl}$ & $\begin{array}{c}\text { C Statistics for Model Predicting } \\
\text { 30-day Readmission/Death, } \\
\text { Including Age, Sex, and Frailty Definition }(95 \% \mathrm{Cl})\end{array}$ \\
\hline CFS (whether they also met the HFRS definition or not) & 2.53 & $1.40-4.57$ & $0.64(0.56-0.70)$ \\
\hline CFS and HFRS & 2.38 & $1.30-4.41$ & $0.60(0.52-0.68)$ \\
\hline CFS only (but not HFRS) & 1.35 & $0.63-2.89$ & $0.54(0.46-0.61)$ \\
\hline HFRS only (but not CFS) & 0.85 & $0.44-1.60$ & $0.55(0.47-0.63)$ \\
\hline \multicolumn{4}{|l|}{ Patients aged $\geq 65$ years } \\
\hline CFS (whether they also met the HFRS definition or not) & 4.31 & $1.80-10.31$ & $0.68(0.59-0.79)$ \\
\hline CFS and HFRS & 2.17 & $0.97-4.83$ & $0.62(0.51-0.72)$ \\
\hline CFS only (but not HFRS) & 2.33 & $0.99-5.47$ & $0.59(0.46-0.71)$ \\
\hline
\end{tabular}

Adjusted odds ratios are for patients meeting the definition of frailty described for that row compared to those not meeting that frailty definition. Abbreviations: CFS, clinical frailty scale; HFRS, hospital frailty risk score.

definitions and 166 (66\%) were frail according to at least one assessment. Agreement between HFRS and the CFS (kappa $0.24,95 \% \mathrm{Cl} 0.16-0.33$ ) was poor. The CFS definition of frailty was $46 \%$ sensitive and $77 \%$ specific in classifying frail patients compared with HFRS-defined frailty.

As we reported earlier, ${ }^{4}$ patients deemed frail were generally similar across scales in that they were older, had more comorbidities, more prescriptions, longer lengths of stay, and poorer quality of life than nonfrail patients (all $P<.01$, Table 1). However, patients classified as frail on the HFRS only but not meeting the CFS definition were younger, had higher quality of life, and despite a similar Charlson Score and number of comorbidities were much more likely to have been living independently prior to admission than those classified as frail on the CFS.

Death or unplanned readmission within 30 days occurred in $13.3 \%$ (65 patients), with most events being readmissions (62, 12.7\%). HFRS-defined frail patients exhibited higher 30-day death/readmission rates (16\% vs $11 \%$ for not frail, $P=.08 ; 14 \%$ vs $11 \%$ in the elderly, $P=.5$ ), which was not statistically significantly different from the nonfrail patients even after adjusting for age and sex (aOR [adjusted odds ratio] 1.62, 95\% $\mathrm{Cl} 0.95-$ 2.75 for all adults; aOR 1.24, 95\% Cl 0.58-2.63 for the elderly). CFS-defined frail patients had significantly higher 30-day readmission/death rates (19\% vs $10 \%$ for not frail, aOR $2.53,95 \% \mathrm{Cl}$ $1.40-4.57$ for all adults and $21 \%$ vs $6 \%$ in the elderly, aOR 4.31 , $95 \% \mathrm{Cl} 1.80-10.31$ ).

Adding the HFRS results to the CFS-based predictive models added little new information, with an integrated discrimination improvement of only 0.009 that was not statistically significant ( $P=.09$, Table 2). In fact, the HFRS was not an independent predictor of postdischarge outcomes after adjusting for age and sex. Although predictive models incorporating the CFS demonstrated the best $\mathrm{C}$ statistics, none of the models had high $C$ statistics (ranging between 0.54 and 0.64 for all adults and between 0.55 and 0.68 for those aged $>65$ years). Even when the frailty definitions were examined as continuous variables, the $\mathrm{C}$ statistics were similar as for the dichotomized analyses ( 0.64 for CFS and 0.58 for HFRS) and the correlation between the two remained weak (Spearman's correlation coefficient 0.34).

\section{DISCUSSION}

We have demonstrated that the prevalence of frailty in patients being discharged from medical wards was high, with the HFRS (44\%) being higher than the CFS (33\%), and that only $46 \%$ of patients deemed frail on the HFRS were also deemed frail on the CFS. We confirm the report by the developers of the HFRS that there was poor correlation between the CFS cumulative deficit model and the administrative-data-based HFRS model in our cohort, even among those older than 65 years.

Previous studies have reported marked heterogeneity in prevalence estimates between different frailty instruments. ${ }^{2,9}$ For example, Aguayo et al. found that the prevalence of frailty in the English Longitudinal Study of Aging varied between $0.9 \%$ and $68 \%$ depending on which of the 35 frailty scales they tested were used, although the prevalence with comprehensive geriatric assessments (the gold standard) was 14.9\% (and $15.3 \%$ on the CFS). ${ }^{9}$ Although frail patients are at higher risk for death and/or readmission after discharge, other investigators have also reported similar findings to ours that frailty-based risk models are surprisingly modest at predicting postdischarge readmission or death, with the $\mathrm{C}$ statistics ranging between 0.52 and 0.57 , although the CFS appears to correlate best with the gold standard of comprehensive geriatric assessment. ${ }^{10-14}$ This is not surprising since the CFS is multidimensional and as a cumulative deficit model, it incorporates assessment of the patient's underlying diseases, cognition, function, mobility, and mood in the assignment of their CFS level. Regardless, others ${ }^{15}$ 
have pointed out the need for studies such as ours to compare the validity of published frailty scales.

Despite our prospective cohort design and blinded endpoint ascertainment, there are some potential limitations to our study. First, we excluded long-term care residents and patients with foreshortened life expectancy - the frailest of the frail - from our analysis of 30-day outcomes, thereby potentially reducing the magnitude of the association between frailty and adverse outcomes. However, we were interested only in situations where clinicians were faced with equipoise about patient prognosis. Second, we assessed only 30-day readmissions or deaths and cannot comment on the impact of frailty definitions on other postdischarge outcomes (such as discharge locale or need for home care services) or other timeframes. Finally, although the association between the HFRS definition of frailty and the 30-day mortality/readmission was not statistically significant, the $95 \%$ confidence intervals were wide and thus we cannot definitively rule out a positive association.

In conclusion, considering that it had the strongest association with postdischarge outcomes and is the fastest and easiest to perform, the most useful of the frailty assessment tools for clinicians at the bedside still appears to be the CFS (both overall and in those patients who are elderly). However, for researchers who are analyzing data retrospectively or policy planners looking at health services data where the CFS was not collected, the HFRS holds promise for risk adjustment in population-level studies comparing processes and outcomes between hospitals.

\section{Acknowledgments \\ The authors would like to acknowledge Miriam Fradette, Debbie Boyko, Sara Belga, Darren Lau, Jenelle Pederson, and Sharry Kahlon for their important contributions in data acquisition in our original cohort study, as well as all the physicians rotating through the general internal medicine wards at the University of Alberta Hospital for their help in identifying the patients. We also thank Dr. Simon Conroy, MB ChB PhD, University of Leicester, UK, for his helpful comments on an earlier draft of this manuscript.}

Disclosures: The authors declare no conflicts of interest. All authors had access to the data and played a role in writing and revising this manuscript.

Funding: Funding for this study was provided by an operating grant from Alberta Innovates - Health Solutions. F.A.M. holds the Chair in Cardiovascular
Outcomes Research at the Mazankowski Heart Institute, University of Alberta. The authors have no affiliations or financial interests with any organization or entity with a financial interest in the contents of this manuscript.

\section{References}

1. Clegg A, Young J, Iliffe S, Rikkert MO, Rockwood K. Frailty in elderly people. Lancet. 2013;381(9868):752-762. doi: 10.1016/S0140-6736(12)62167-9.

2. Collard RM, Boter H, Schoevers RA, Oude Voshaar RC. Prevalence of frailty in community-dwelling older persons: a systematic review. J Am Geriatr Soc. 2012;60(8):1487-1492. doi: 10.1111/j.1532-5415.2012.04054.x.

3. de Vries NM, Staal JB, van Ravensberg CD, Hobbelen JS, Olde Rikkert MG, Nijhuis-van der Sanden MW. Outcome instruments to measure frailty: a systematic review. Ageing Res Rev. 2011;10(1):104-114. doi: 10.1016/j. arr.2010.09.001.

4. Belga S, Majumdar SR, Kahlon S, et al. Comparing three different measures of frailty in medical inpatients: multicenter prospective cohort study examining 30-day risk of readmission or death. J Hosp Med. 2016;11(8):556-562. doi: 10.1002/jhm.2607.

5. Rockwood K, Andrew M, Mintnitski A. A comparison of two approaches to measuring frailty in elerly people. J Gerontol. 2007;62(7):738-743. doi: 10.1093/gerona/62.7.738.

6. Gilbert T, Neuburger J, Kraindler J, et al. Development and validation of a Hospital Frailty Risk Score focusing on older people in acute care settings using electronic hospital records: an observational study. Lancet. 2018;391(10132):1775-1782. doi: 10.1016/S0140-6736(18)30668-8Get.

7. Kahlon S, Pederson J, Majumdar SR, et al. Association between frailty and 30-day outcomes after discharge from hospital. CMAJ. 2015;187(11):799-804. doi: 10.1503/cmaj.150100.

8. Pencina MJ, D' Agostino RB, Vasan RS. Evaluating the added predictive ability of a new marker: from area under the roc curve to reclassification and beyond. Stat Med. 2008;27(2):157-172. doi: 10.1002/sim.2929.

9. Aguayo GA, Donneau A-F, Vaillant MT, et al. Agreement between 35 published frailty scores in the general population. Am J Epidemiol. 2017;186(4):420-434. doi: 10.1093/aje/kwx061.

10. Ritt M, Bollheimer LC, Siever CC, Gaßmann KG. Prediction of one-year mortality by five different frailty instruments: a comparative study in hospitalized geriatric patients. Arch Gerontol Geriatr. 2016;66:66-72. doi: 10.1016/j.archger.2016.05.004

11. Forti P, Rietti E, Pisacane N, Olivelli V, Maltoni B, Ravaglia G. A comparison of frailty indexes for prediction of adverse health outcomes in a elderly cohort. Arch Gerontol Geriatr. 2012;54(1):16-20. doi: 10.1016/j.archger.2011.01.007.

12. Wou F, Gladman JR, Bradshaw L, Franklin M, Edmans J, Conroy SP. The predictive properties of frailty-rating scales in the acute medical unit. Age Ageing. 2013;42(6):776-781. doi: 10.1093/ageing/aft055.

13. Wallis SJ, Wall J, Biram RW, Romero-Ortuno R. Association of the clinical frailty scale with hospital outcomes. QJM. 2015;108(12):943-949. doi: 10.1093/qjmed/hcv066.

14. Harmand MGC, Meillon C, Bergua V, et al. Comparing the predictive value of three definitions of frailty: results from the Three-City Study. Arch Gerontol Geriatr. 2017;72:153-163. doi: 10.1016/j.archger.2017.06.005

15. Bouillon K, Kivimaki M, Hamer M, et al. Measures of frailty in population-based studies: an overview. BMC Geriatrics. 2013;13(1):64. doi: 10.1186/1471-2318-13-64. 